\title{
Dissolution Model Development: Formulation Effects and Filter Complications
}

\author{
Ragna Berthelsen ${ }^{1}$, René Holm², Jette Jacobsen ${ }^{1}$, Jakob Kristensen ${ }^{3}$, \\ Bertil Abrahamsson ${ }^{4}$, and Anette Müllertz ${ }^{1,5, *}$ \\ ${ }^{1}$ Department of Pharmacy, University of Copenhagen, Denmark \\ ${ }^{2}$ Biologics and Pharmaceutical Sciences, H. Lundbeck A/S, Copenhagen, Denmark \\ ${ }^{3}$ cJakob Pharma, Seattle, WA, USA \\ ${ }^{4}$ AstraZeneca Pharmaceutics, R\&D, Mölndal, Sweden \\ ${ }^{5}$ Bioneer: FARMA, Department of Pharmacy, University of Copenhagen, Denmark
}

e-mail: Anette.mullertz@sund.ku.dk

\begin{abstract}
This study describes various complications related to sample preparation (filtration) during development of a dissolution method intended to discriminate among different fenofibrate immediate-release formulations. Several dissolution apparatus and sample preparation techniques were tested. The flow-through cell apparatus (USP 4) was found unfit for dissolution testing of fenofibrate MeltDose formulations due to clogging of filters and varying flow rates. A mini paddle dissolution setup produced dissolution profiles of the tested formulations that correlated well with clinical data. The work towards the mini paddle dissolution method demonstrates that sample preparation influenced the results. The investigations show that excipients from the formulations directly affected the drug-filter interaction, thereby affecting the dissolution profiles and the ability to predict the in vivo data. With the tested drug-formulation combination, the best in vivo-in vitro correlation was found after filtration of the dissolution samples through $0.45-\mu \mathrm{m}$ hydrophobic PTFE membrane filters.
\end{abstract}

KEYWORDS: Dissolution; USP Apparatus 2; USP Apparatus 4; IVIVC; drug-filter interaction; fenofibrate.

\section{INTRODUCTION}

D issolution testing is a very important in vitro test utilized for several different purposes in pharmaceutical drug development and as a quality control tool to monitor batch-to-batch consistency of drug release from a dosage form. Additionally, it is often used as an in vitro surrogate for in vivo performance that can guide formulation development (1). In the formulation development phase, dissolution tests are used to validate initial screening among potential formulations and to help in the selection of the candidate formulation. To discriminate between formulations producing different in vivo outcomes, the dissolution method needs to predict the in vivo situation generating an in vivo-in vitro correlation (IVIVC).

Several aspects are important to consider in the development of an appropriate dissolution method for a given active pharmaceutical ingredient (API) and drug formulation. Among others, the choice of apparatus, dissolution medium, and sample preparation are all factors that will directly impact the dissolution profiles (2-4).

The present study describes various complications related to sample preparation (filtration) during development of a dissolution method intended for discrimination between different immediate-release formulations containing the poorly water soluble drug fenofibrate. During the dissolution method development, several dissolution apparatus, dissolution media, and sample preparation techniques were tested.

\section{MATERIALS AND METHODS \\ Chemicals}

Fenofibrate was purchased from Sigma-Aldrich Chemie (Deisenhofen, Germany). MeltDose (MD) formulations $A, B$, and $C$ as well as the physical mixture of formulation A were kindly provided by LifeCyclePharma (Hørsholm, Denmark). Sodium hydroxide and sodium chloride were from Merck (Darmstadt, Germany), and methanol (isocratic grade) was purchased from VWR International (Lutterworth, UK). Potassium dihydrogen phosphate (ACS grade), crude porcine bile extract, and maleic acid (analytical grade) were purchased from Sigma-Aldrich Chemie (St. Louis, MO, USA), and phosphatidyl choline from soybean lecithin (Lipoid S PC) was from Lipoid GmbH (Ludwigshafen, Germany). Purified water was obtained from a Milli-Q water purification system (Millipore, Bedford, Massachusetts). 


\section{Model Drug and Formulations Employed}

Fenofibrate is a poorly water soluble compound with an aqueous saturation solubility of less than $1 \mu \mathrm{g} / \mathrm{mL}(5-7)$ and a $\log P$ of 5.2 (8). In the three tested formulations, fenofibrate was dissolved with different surfactants in a melted hydrophilic polymer (vehicle temperature $<79.5$ ${ }^{\circ} \mathrm{C}$ ) that was sprayed onto inert particles forming granula ready for direct compression (MeltDose technique) (9). Table 1 shows the surfactant composition of the tested formulations. More details on the formulation contents as well as human clinical data on the absorption of fenofibrate after oral administration of the three MeltDose (MD) formulations, are available in the literature (10). The area under the curve (AUC) of the plasma concentrationtime curves and the maximum plasma concentration $\left(C_{\max }\right)$ both had the rank order $A>B>C$ for the three formulations (10).

Table 1. Surfactant Composition of the MD Tablet Formulations

\begin{tabular}{|l|l|c|l|c|}
\hline \multirow{2}{*}{ Formulation } & \multicolumn{2}{|c|}{ Surfactant 1 } & \multicolumn{2}{c|}{ Surfactant 2 } \\
\cline { 2 - 5 } & Name & $\begin{array}{c}\text { Amount } \\
\text { (mg/tablet) }\end{array}$ & Name & $\begin{array}{c}\text { Amount } \\
\text { (mg/tablet) }\end{array}$ \\
\hline A (MD and PM) & Poloxamer 407 & 53.8 & - & - \\
\hline B (MD) & Poloxamer 188 & 16.2 & Cremophor ELP & 37.7 \\
\hline C (MD) & Poloxamer 188 & 53.8 & SLS & 19.9 \\
\hline
\end{tabular}

A physical mixture (PM) of formulation A was included in selected studies to determine the effect of the formulation principle in the dissolution models. The PM contained the same amount of fenofibrate as the MD A formulation and was prepared by mixing crystalline fenofibrate with a placebo granule prepared in the same manner as formulation A. The PM of fenofibrate and placebo granule was compressed into tablets similar to the MD formulations.

\section{Dissolution Studies}

USP 4: Flow-Through Cell Apparatus

With a setup ensuring sink conditions, dissolution studies were performed using the flow-through cell (FTC) dissolution apparatus (USP Apparatus 4) consisting of an automated system (Dissotest CE70, Sotax, Basel, Switzerland) with a fraction collector and a medium splitter. Sample fractions were collected at predetermined intervals $(5,10,15,20,25,30,45,60 \mathrm{~min})$. The equipment was fitted with 22.6-mm cells (internal diameter) and the temperature kept at $37.0 \pm 0.5^{\circ} \mathrm{C}$. A ruby ball $(5-\mathrm{mm}$ diam) and $6.5 \mathrm{~g}$ of glass beads (1-mm diam) were placed in the bottom of the dissolution cell cone to ensure laminar flow of fluid entering the cell. Fenofibrate MD and PM tablets (145-mg dose) were placed on top of the glass beads. A tablet holder prevented the tablet from floating. Undissolved fenofibrate particles were held back by a glass-fiber filter (GD-120, 2.7- $\mu \mathrm{m}$ pore size, $25-\mathrm{mm}$ diam, Advantec, Tokyo Roshi Kaisha Ltd., Japan) fixed at the top of the dissolution flow cells. The flow rate was $10.0 \pm 0.5$ $\mathrm{mL} / \mathrm{min}$, and the apparatus was operated in an openloop mode. All experiments were performed in at least triplicate. Due to the large volumes of dissolution media needed, initial FTC dissolution studies were performed with simple media comprising degassed purified water or degassed phosphate buffer (50 mM) pH 6.8.

The flow rate was set manually and verified by measurements of media running through empty FTCs. As a method control, the flow rate was measured during the first $25 \mathrm{~min}$ of each dissolution run. Every sample collected was weighed, and the flow rate was calculated as the mass of the medium that passed through the FTC per time unit.

\section{USP 2: Paddle Dissolution}

To simulate the condition in the upper small intestine, Copenhagen fasted-state simulated intestinal media ( $\mathrm{CPH}$ fasted) was used. Medium composition and preparation has previously been described (11). In short, CPH fasted contained $2.5 \mathrm{mM}$ bile salts and $0.625 \mathrm{mM}$ phospholipid in a $20 \mathrm{mM}$ maleic acid buffer, $\mathrm{pH}$ 6.5. The osmolality was adjusted to $270 \mathrm{mOsm} / \mathrm{kg}$ by the addition of sodium chloride.

Dissolution tests of the three fenofibrate MD formulations in $\mathrm{CPH}$ fasted were carried out using standard dissolution volumes of 500 or $1000 \mathrm{~mL}$ per vessel. All experiments were conducted at $37 \pm 0.5{ }^{\circ} \mathrm{C}$ and a paddle rotation speed of $50 \mathrm{rpm}$. Plastic syringes $(5 \mathrm{~mL})$ connected to a stainless steel sampling device were used for manual sampling at fixed sampling times $(2,4,6,8,10,15,30$, and $60 \mathrm{~min}$ ). Sample preparation consisted of a single filtration step discarding the first $1 \mathrm{~mL}$ followed by centrifugation for $10 \mathrm{~min}$ at 15,000 rpm and appropriate dilution before analysis by high performance liquid chromatography (HPLC). Syringe filters with two different types of membranes were tested: $0.45-\mu$ m hydrophobic polytetrafluoroethylene (PTFE) and $0.45-\mu \mathrm{m}$ cellulose acetate (CA) (both from VWR International, Radnor, PA, USA). Immediately after sampling, the sample volume was replaced with fresh dissolution medium at $37^{\circ} \mathrm{C}$.

Dissolution studies of the fenofibrate MD formulations were also carried out using a mini paddle assembly with special inserts and $250-\mathrm{mL}$ vessels (Erweka DT 70, Heusenstamm, Germany), representing a scaled-down version of USP Apparatus 2 (12). In this apparatus, 
dissolution tests were performed with 75 or $100 \mathrm{~mL}$ dissolution medium (CPH fasted). The sample size was 2 $\mathrm{mL}$, and the stirring speed $75 \mathrm{rpm}$; all other parameters were as described for the standard USP Apparatus 2. All dissolution studies were performed in triplicate or quadruplicate.

\section{Fenofibrate Equilibrium Solubility}

The solubility of fenofibrate was determined in all tested dissolution media. Fenofibrate was added in excess and incubated with $10 \mathrm{~mL}$ of medium on an end-to-end rotator at $37^{\circ} \mathrm{C}$. After 2, 5, 24, and $48 \mathrm{~h}$, the test tubes were removed from the rotator and centrifuged at 4,500 $\mathrm{rpm}$ for $10 \mathrm{~min}$. Following centrifugation, a 1-mL sample was transferred to an Eppendorf tube and centrifuged at $15,000 \mathrm{rpm}$ for $10 \mathrm{~min}$ so that all undissolved drug was spun down in a pellet. The test tubes was shaken vigorously and placed back on the rotator at $37^{\circ} \mathrm{C}$. The supernatant in the Eppendorf tube was diluted appropriately and analyzed by HPLC. The study was performed in triplicate.

\section{Filter Adsorption}

As a control study, drug adsorption to the different types of filters was determined. Fenofibrate was dissolved in $\mathrm{CPH}$ fasted in a concentration corresponding to $80 \%$ of the saturation solubility $(8.7 \mu \mathrm{g} / \mathrm{mL})$. The concentration of fenofibrate in this solution was analyzed by HPLC without filtration as well as after filtration through either of the tested filters described for the dissolution studies (CA or PTFE membranes). With filtration, the first $1 \mathrm{~mL}$ was discarded.

\section{Quantitative Analysis of Fenofibrate}

All samples were analyzed using an Agilent 1100 HPLC system (1100 autosampler, 1100 quaternary pump with degasser, 1100 thermostatted column compartment, and 1100 diode array detector) (Agilent, Palo Alto, CA). A Luna C18 column $(10 \mu \mathrm{m}, 3.9 \times 300 \mathrm{~mm})$, protected with a guard column (Phenomex C18, $4 \times 3 \mathrm{~mm}$ ) was used as the stationary phase and maintained at $40{ }^{\circ} \mathrm{C}$ during analysis. Methanol and purified water in the ratio 85:15 was used as the mobile phase. The flow rate was controlled at $1.2 \mathrm{~mL} / \mathrm{min}$. The effluent was monitored at $280 \mathrm{~nm}$ for $12 \mathrm{~min}$, and the drug retention time was 9-10 min. The injection volume was $20 \mu \mathrm{L}$.

\section{Data Analysis}

Single time point comparisons of the amount of fenofibrate dissolved were done using a two-way ANOVA test, followed by pair-wise comparison of means using the Tukey test $(\alpha=0.05)$. All data analysis was performed using GraphPad Prism, 6.0 (GraphPad Software, San Diego, CA, USA).

\section{RESULTS AND DISCUSSION}

\section{Flow-Through Cell Dissolution}

The FTC method holds some advantages over standard dissolution methods such as paddle and basket. Some of the distinct advantages of the FTC apparatus include easy medium and flow rate changes within a single run, identification of carry-over effects, and easier in vitroin vivo correlations because a single dissolution profile corresponding to the drug release through the entire gastrointestinal lumen is obtainable. Another prominent advantage is that the open-loop configuration of the FTC system is effective for providing sink conditions. Consequently, this method has been particularly suggested for dissolution tests of poorly soluble compounds (13). Among the disadvantages of the FTC apparatus are a risk of filter clogging, difficulties when validating the flow rate during testing, and a requirement for very large volumes of medium for open-system runs (14). Because of the many advantages, especially for poorly water soluble compounds, the FTC apparatus was the first choice in the present dissolution method development process.

As a control parameter, the flow rate was monitored manually during the FTC experiments. Figure 1 shows the mean mass flow rate measured in 5-min intervals over the first 25 min of four different experiments. The control, flow cells without a formulation, showed a constant flow with only small variations well within the USP criteria (nominal flow rate $\pm 5 \%$ ) (15). The flow measurements revealed that the fenofibrate MD formulations interrupted the flow rate to a very large and very varying extent. Within a single run, flow rates varied with standard deviations (SD) up to $50 \%$. In particular, formulation A, containing poloxamer 407 as the main surfactant, disrupted the flow substantially, decreasing it to $3.5 \pm 2.4 \mathrm{~g} / \mathrm{mL}$ after 25 min of dissolution. In contrast, the physical mixture of formulation A did not seem to disrupt the flow at all, and the average flow rate was $9.9 \pm 0.3 \mathrm{~g} / \mathrm{mL}$. The physical mixture of formulation A contained exactly the same amounts of drug and excipients, but bulk fenofibrate was simply mixed with the placebo granular of formulation A before tableting instead of incorporating fenofibrate in the MD process. Poloxamer 407 is a thermosensitive polymer that forms a gel at temperatures above $20^{\circ} \mathrm{C}(16)$. If a gel forms within the FTC as a result of the warm dissolution medium (37 ${ }^{\circ} \mathrm{C}$ ), it might block the filter. However, because the PM mixture of formulation A containing the same amount of poloxamer 407 did not disrupt the flow rate, this suggested explanation was uncertain. The difference in fenofibrate particle size might also explain the difference in flow rate due to filter clogging by the small fenofibrate particles in the MD formulation. However, because formulations $B$ 
and $C$ containing fenofibrate with a particle size similar to that of formulation $A(1 \mu \mathrm{m})$ did not disrupt the flow rate significantly ( $p>0.05$ for all samples compared with the control), this could not be the only reason.

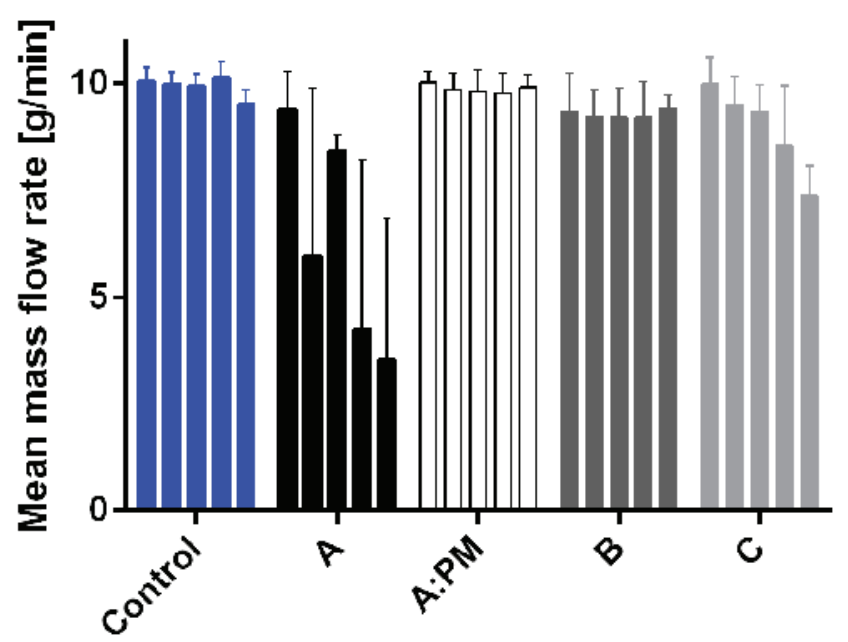

Figure 1. Mean mass flow rate during flow-through dissolution of four different fenofibrate formulations and a control. Each subcolumn represents a 5-min interval. Error bars represent mean $\pm S D(n=4)$. USP criterion was $\pm 5 \%$ of the nominal flow rate.

To ensure efficient filtration and avoid backpressure in the FTC apparatus, appropriate filter selection is required. The most common type of filter used in the FTC dissolution apparatus is glass-fiber filter (14). The filters utilized in the present study were glass-fiber filters with a nominal poor size of $2.7 \mu \mathrm{m}$. They were chosen based on prior experience with the FTC apparatus and poorly water soluble compounds $(17,18)$. To avoid the aforementioned filter-clogging problem, a switch to filters with a larger pore size was considered. However, preceding this consideration, the chosen filters were evaluated for their capability of retaining fenofibrate in the tested method setup. Before HPLC analysis, one set of samples from formulation $A$ (MD and $P M$ ) was diluted 1:1 with mobile phase, while another set was analyzed directly. Analysis results show that approximately five times less fenofibrate was solubilized from all the undiluted samples (17.8 \pm $3.4 \%$ compared with the diluted samples), indicating that the filters did not retain all undissolved fenofibrate within the FTCs from either formulation. To retain more fenofibrate during the dissolution process, filters with a smaller pore sizes could be used; however, because a smaller pore size would presumably add back pressure causing even more clogging, this option was abandoned. A more detailed study on filter selection including alternative filters, combinations of filters, and inclusion of glass wool, for example (14), may have solved the clogging issues. However, in the present method development, the tested glass-fiber filters were not appropriate with these fenofibrate formulations. Because flow rate is one of the most critical factors for the FTC apparatus (19), the observed flow variations led to a discontinuation of method development using this apparatus.

\section{Paddle Dissolution}

As alternative dissolution apparatus, the standard USP Apparatus 2 and a miniaturized version of the same apparatus were tested. With these apparatus, a series of experiments were performed with varying dissolution volumes (500 and $1000 \mathrm{~mL}$ using the standard setup, and $75 \mathrm{~mL}$ and $100 \mathrm{~mL}$ using the mini paddle setup), varying paddle speed (50 and $75 \mathrm{rpm}$ ), and different sample preparations (filtration through CA and PFTE membrane filters). These studies led to the development of a dissolution model producing dissolution profiles for the three MD formulations correlating with the clinical data (10). Dissolution data using $100 \mathrm{~mL}$ and $1000 \mathrm{~mL}$ $\mathrm{CPH}$ fasted medium, $75 \mathrm{rpm}$, and $0.45-\mu \mathrm{m}$ PTFE filters were described previously alongside the clinical data by Berthelsen et al. (10) as part of a different study; data from studies using $75 \mathrm{~mL}$ and $500 \mathrm{~mL}$ dissolution medium or 50 rpm are not shown. In short, the successful method was based on the miniaturized paddle dissolution apparatus using $100 \mathrm{~mL}$ of CPH fasted and a paddle speed of $75 \mathrm{rpm}$. When testing different filter types for sample preparation purposes, some interesting observations were made. Figure 2 shows the dissolution profiles of the three tested MD formulations each containing $145 \mathrm{mg}$ fenofibrate per tablet using the mini paddle apparatus $(100 \mathrm{~mL} \mathrm{CPH}$ fasted-state media, $75 \mathrm{rpm}, 37^{\circ} \mathrm{C}$ ) obtained using two different types of filters (CA or PTFE membrane). The results show clear differences in the dissolution profiles obtained for samples prepared with different filters. For all formulations, the use of CA filters led to a decreased amount of drug dissolved from all formulations compared with PTFE filters. For each formulation, the difference in the amount of fenofibrate dissolved after $60 \mathrm{~min}$ was $60 \%, 75 \%$, and $60 \%$ for formulations $A, B$, and C, respectively (CA versus PTFE). The main reason for these results is probably that fenofibrate adsorbs differently to the two evaluated filters, which is a common problem discussed in other publications $(20,21)$. Dressman and coworkers (20) have shown that another poorly water soluble drug, griseofulvin, dissolved in simulated intestinal fluid adsorbed to similar CA filters producing a recovery in the filtered samples of $73.7 \pm 2.3 \%$ and $71.6 \pm 3.6 \%$, with and without discarding the first $2 \mathrm{~mL}$ of each sample, respectively. In the present study, the adsorption of fenofibrate to the different filters was tested. The results, shown in Figure 3, confirm that fenofibrate adsorbed 
to CA membrane filters to a much higher degree than to PFTE membrane filters, $49.1 \pm 8.2 \%$ vs $93.8 \pm 3.2 \%$ drug recovery. This high degree of drug adsorption to the CA membrane filters correlates well with the fact that fenofibrate barely reached its saturation solubility in the pure dissolution medium (not accounting for the solubilizing effects of the formulation surfactants) when using the CA filters (Figure 2). Because of this high degree of drug adsorption to CA filters, they should not be used in dissolution testing of poorly water soluble drugs.

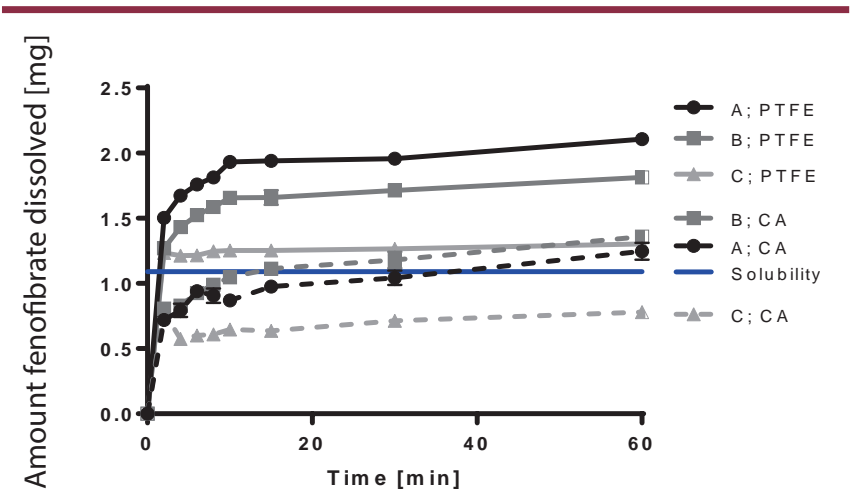

Figure 2. Dissolution profiles of fenofibrate MeltDose formulation $A(\bullet)$, formulation $B(\square)$, and formulation $C(\triangle)$ using PTFE membrane filters (full lines) or CA membrane filters (dotted lines). The amount of fenofibrate dissolvable in the pure dissolution medium determined in a separate solubility study is indicated by the blue line. Values are mean $\pm S D(n=3)$.

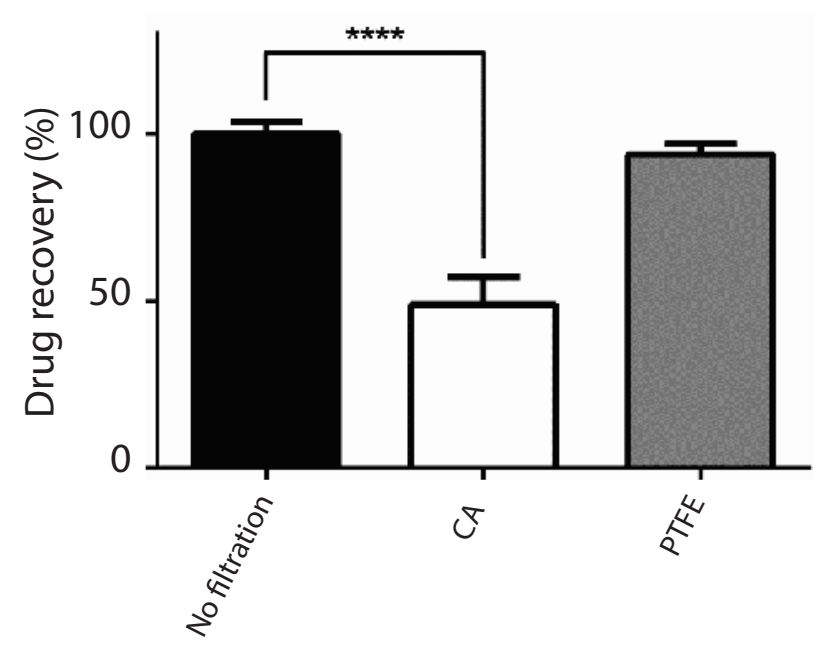

Figure 3. Fenofibrate relative drug recovery from samples of a fenofibrate solution filtrated through CA and PTFE membrane filters compared with unfiltered samples $(n=3)$. ( **** indicates a significant difference with $p<0.0001$.)

For the present dissolution method development, the choice of filters was obvious based on the adsorption study. However, the dissolution profiles in Figure 2 show that a change in filter type for sample preparation resulted in a different rank order of the amount of fenofibrate dissolved from the three MD formulations after 60 min of dissolution. The rank orders, as illustrated in Figure 2, are: $B>A>C$ with $C A$ filters and $A>B>C$ with PTFE filters, with a statistically significant difference $(p<0.05)$ between the amount of fenofibrate dissolved after 60 min from all formulations compared with one another (using the same type of filter). The change in rank ordering of the formulations has a massive impact on the IVIVC for the formulations (i.e., the choice of filters might dictate which formulation is expected to produce the highest $C_{\max }$ or $A U C$ ). A comparison of the dissolution profiles with the clinical data of the formulations shows that the dissolution results obtained after filtration with the PTFE filters gave the most reliable results (10).

The switch in rank order could only be attributed to a difference in the filter interaction of the drug and surfactant combinations, because the sole difference between the three formulations was the choice and amount of surfactants. CA filters are hydrophilic, whereas PTFE filters are hydrophobic. Fenofibrate is a neutral compound and is expected to absorb more to hydrophobic surfaces. However, because the formulations contained different surfactants, different micelles were formed in the dissolution medium. These micelles would encapsulate the lipophilic fenofibrate while presenting a hydrophilic surface toward the aqueous environment, resulting in higher adsorption to the CA filters than to the PTFE filters.

Formulation $B$ adsorbed less to the CA filters than formulations $\mathrm{A}$ and $\mathrm{C}$, which correlates with the formulations tendency to disrupt the flow rate in the FTC apparatus. Based on these results, it might be speculated that Cremophor ELP, which is a nonionic surfactant only present in formulation $\mathrm{B}$, contributes to the formation of micelles that adsorb less to different types of filters than the surfactants included in the two other formulations. The observation that the formulation affected the drug-filter interactions shows that a simple adsorption study only accounting for drug adsorption in the absence of formulation will possibly not produce enough information. Still, in the present case where the hydrophobic PTFE membrane filters led to the lowest drug adsorption and the right formulation rank order, the adsorption study led to the choice of the right filters for the sample preparation.

The present study demonstrates the potential variables in the development of an in vitro dissolution method. The results further demonstrate the importance of obtaining clinical data to support the development work, which for 
difficult compounds could even be designed to ensure the development of a relevant in vitro method able to discriminate among similar drug formulations.

\section{CONCLUSION}

The present study underlines the importance of choosing the right apparatus and sample preparation when developing a new dissolution method for a given drug compound and drug formulation. For fenofibrate MD formulations, the FTC apparatus with the tested glassfiber filter was unfit for dissolution analysis due to clogging of filters and induction of variable flow rates. A mini paddle dissolution setup produced dissolution profiles of the three fenofibrate MD formulations that correlated with the clinical data. However, the sample preparation (i.e., filtration) had a large impact on the obtained results. The present study therefore stresses the importance of obtaining an IVIVC when developing a new dissolution method, as the lack of relevant in vivo data for evaluation may lead to mistaken conclusions regarding the method and erroneous predictions of in vivo outcome.

Furthermore, this study shows that formulation excipients can affect the drug-filter interaction/adsorption, directly affecting the dissolution profiles and the prediction of in vivo data. With the tested drug-formulation combination, the best IVIVC was found after filtration of the dissolution samples through 0.45- $\mu \mathrm{m}$ hydrophobic PTFE membrane filters.

\section{FUNDING}

This work has been supported by the Predicting Drug Absorption Innovation Consortium (PDA).

\section{CONFLICT OF INTEREST}

No conflict of interest has been declared by the authors.

\section{REFERENCES}

1. Dissolution Testing of Immediate Release Solid Oral Dosage Forms; Guidance for Industry; U.S. Department of Health and Human Services, Food and Drug Administration, Center for Drug Evaluation and Research (CDER), U.S. Government Printing Office: Washington, DC, 1997.

2. Chevalier, E.; Viana, M.; Artaud, A.; Chomette, L.; Haddouchi, S.; Devidts, G.; Chulia, D. Comparison of Three Dissolution Apparatuses for Testing Calcium Phosphate Pellets used as Ibuprofen Delivery Systems. AAPS PharmSciTech 2009, 10 (2), 597-605. DOI: 10.1208/s12249-009-9252-z.

3. Emara, L. H.; Taha, N. F.; Mursi, N. M. Investigation of the Effect of Different Flow-Through Cell Designs on the Release of Diclofenac Sodium SR Tablets.
Dissolution Technol. 2009, 16 (2), 23-31. DOI: 10.14227/DT160209P23.

4. Fotaki, N.; Aivaliotis, A.; Butler, J.; Dressman, J.; Fischbach, M.; Hempenstall, J.; Klein, S.; Reppas, C. A comparative study of different release apparatus in generating in vitro-in vivo correlations for extended release formulations. Eur. J. Pharm. Biopharm. 2009, 73 (1), 115-120. DOI: 10.1016/j.ejpb.2009.04.012.

5. Hanafy, A.; Spahn-Langguth, H.; Vergnault, G.; Grenier, P.; Tubic Grozdanis, M.; Lenhardt, T.; Langguth, P. Pharmacokinetic evaluation of oral fenofibrate nanosuspensions and SLN in comparison to conventional suspensions of micronized drug. Adv. Drug Delivery Rev. 2007, 59 (6), 419-426. DOI: 10.1016/j.addr.2007.04.005.

6. Law, D.; Wang, W.; Schmitt, E. A.; Qiu, Y.; Krill, S. L.; Fort, J. J. Properties of rapidly dissolving eutectic mixtures of poly(ethylene glycol) and fenofibrate: The eutectic microstructure. J. Pharm. Sci. 2003, 92 (3), 505-515. DOI: 10.1002/jps.10324.

7. Vogt, M.; Kunath, K.; Dressman, J. B. Dissolution enhancement of fenofibrate by micronization, cogrinding and spray-drying: Comparison with commercial preparations. Eur. J. Pharm. Biopharm. 2008, 68 (2), 283-288. DOI: 10.1016/j. ejpb.2007.05.010.

8. Persson, L. C.; Porter, C. J.; Charman, W. N.; Bergström, C. A. Computational Prediction of Drug Solubility in Lipid Based Formulation Excipients. Pharm. Res. 2013, 30 (12), 3225-3237. DOI: 10.1007/s11095-0131083-7.

9. Holm, P.; Norling, T. A Tablet Comprising a Fibrate. International patent WO2006084475 A2, August 17, 2006.

10. Berthelsen, R.; Sjögren, E.; Jacobsen, J.; Kristensen, J.; Holm, R.; Abrahamsson, B.; Müllertz, A. Combining in vitro and in silico methods for better prediction of surfactant effects on the absorption of poorly water soluble drugs-a fenofibrate case example. Int. J. Pharm. 2014, 473 (1-2), 356-365. DOI: 10.1016/j. ijpharm.2014.06.060.

11. Kleberg, K.; Jacobsen, J.; Müllertz, A. Characterising the behaviour of poorly water soluble drugs in the intestine: application of biorelevant media for solubility, dissolution and transport studies. J. Pharm. Pharmacol. 2010, 62 (11), 1656-1668. DOI: 10.1111/j.2042-7158.2010.01023.x.

12. Klein, S.; Shah, V. P. A Standardized Mini Paddle Apparatus as an Alternative to the Standard Paddle. AAPS PharmSciTech 2008, 9 (4), 1179-1184. DOI: 10.1208/s12249-008-9161-6.

13. Fotaki, N.; Reppas, C. The Flow Through Cell 
Methodology in the Evaluation of Intralumenal Drug Release Characteristics. Dissolution Technol. 2005, 12 (2), 17-21. DOI: 10.14227/DT120205P17.

14. Fotaki, N. Flow-Through Cell Apparatus (USP Apparatus 4): Operation and Features. Dissolution Technol. 2011, 18 (4), 46-49. DOI: 10.14227/ DT180411P46.

15. The United States Pharmacopeia and National Formulary USP 35-NF 30; The United States Pharmacopeial Convention, Inc.: Rockville, MD, 2012.

16. Cespi, M.; Bonacucina, G.; Pucciarelli, S.; Cocci, P.; Perinelli, D. R.; Casettari, L.; Illum, L.; Palmieri, G. F.; Palermo, F. A.; Mosconi, G. Evaluation of thermosensitive poloxamer 407 gel systems for the sustained release of estradiol in a fish model. Eur. J. Pharm. Biopharm. 2014, 88 (3), 954-961. DOI: 10.1016/j.ejpb.2014.08.008.

17. Sunesen, V. H.; Pedersen, B. L.; Kristensen, H. G.; Müllertz, A. In vivo in vitro correlations for a poorly soluble drug, danazol, using the flow-through dissolution method with biorelevant dissolution media. Eur. J. Pharm. Sci. 2005, 24 (4), 305-313. DOI: 10.1016/j.ejps.2004.11.007.

18. Thybo, P.; Pedersen, B. L.; Hovgaard, L.; Holm, R.; Müllertz, A. Characterization and Physical Stability of Spray Dried Solid Dispersions of Probucol and PVP-K30. Pharm. Dev. Technol. 2008, 13 (5), 375-386. DOI: $10.1080 / 10837450802244843$.

19. Gray, V.A. Compendial Testing Equipment: Calibration, Qualification, and Sources of Error. In Pharmaceutical Dissolution Testing; Dressman, J. B., Krämer, J., Eds.; Taylor \& Francis Group: Boca Raton, FL, 2005; pp 3967. DOI: $10.1201 / 9780849359170 . c h 2$.

20. Lindenberg, M.; Wiegand, C.; Dressman, J. B. Comparison of the Adsorption of Several Drugs to Typical Filter Materials. Dissolution Technol. 2005, 12 (1), 22-25. DOI: 10.14227/DT120105P22.

21. Joshi, V.; Blodgett, J.; George, J.; Brinker, J. Impact of Sample Preparation on Dissolution Testing: Drug Binding and Extracable Impurities and Their Effect on Dissolution Data. Dissolution Technol. 2008, 15 (4), 20-27. DOI: 10.14227/DT150408P20. 